\title{
PhILOSOPHIE ET AUTORITE LOGIQUE: LA QUESTION VITALE POUR LA PHILOSOPHIE FRANÇAISE
}

Philosophy AND LOGICAL AUTHORITY: THE VITAL QUESTION FOR FRENCH PHILOSOPHY.

Jeronimo Milone

École Normale Supérieure

Paris, França

ORCID 0000-0003-4105-6767

\section{Résumé}

Cet article propose la présentation de quelques traits particuliers de la philosophie française au XXème siècle. S'appuyant aussi sur des considérations de Fréderic Worms, on lira ici le jeune Derrida et ses influences afin de determiner comment une discussion sur la vie et les aspects biologiques de la pensée auront marqué le profil de la philosophie française lui donnant sa caractéristique toute particulière. Les questions posées ici, alors, se doivent à l'effort de penser comment et pourquoi l'autorité logique, mathématique et, par extension, scientifique, s'appuie sur des arguments qui convoquent la vie, l'essence du vivant, pour conformer la légitimité de cette autorité. Ce sont donc les déploiements de la phénoménologie husserlienne dans l'œuvre de Cavaillès les aspects qui se rassembleront dans la pensée de Derrida, pour qui la question de la mort et de la sexualité a été toujours centrale.

Mots-clés: Derrida; Cavaillès; Vie; Logique.

\section{Resumo}

Este artigo propóe a apresentação de alguns traços particulares $\mathrm{da}$ filosofia francesa do século XX. Apoiando-se também nas consideraçóes Frédéric Worms, far-se-á uma leitura do jovem Derrida et das suas influências, a fim de sublinhar de que modo uma discussão sobre a vida e os aspectos biológicos do pensamento terão marcado o perfil da filosofia francesa, conferindo-lhe, e não outra coisa, sua característica muito singular. As questóes dispostas aqui, assim, devem-se

\section{Abstract}

This paper undertakes to delineate some important characteristics of 20thcentury French philosophy. On the basis of the work of Frédéric Worms, we propose to read the young Derrida and his influences so as to show how the debate around the idea of life and the biological features of thought defines the profile of French philosophy. Indeed, it is this debate, and not some other thing, that determines its peculiarity. The questions that we approach derive from the task of thinking how 
ao esforço de pensar como e por que a autoridade lógica, matemática e, por extensão, científica, apoia-se sobre argumentos que convocam a vida, a essência do vivo, para conformar a legitimidade da desta autoridade. São, portanto, os desenvolvimentos da fenomenologia husserliana na obra de Cavaillès, os aspectos que se agruparão no pensamento de Derrida, para quem a questáo da morte e da sexualidade sempre foi central.

Palavras-chave: Derrida; Cavaillès; Vida; Lógica. and why logical, mathematical, and by extension, scientific authority is grounded - so as to establish its legitimacy - on arguments that refer to life, and to the essence of living. The developments of the Husserlian phenomenology in the works of Cavaillès are, in particular, the aspects that will frame Derrida's thought, for whom the question of death and sexuality was always central.

Keywords: Derrida; Cavaillès; Life; Logic.

La vida es demasiado pobre para no ser también inmortal. (BORGES, Sentirse en muerte in: El idioma de los argentinos)

Sabemos que era enemigo de los filósofos; apoderarse de una de sus armas y volverla contra ellos, debió causarle un belicoso placer. (BORGES, Historia de la eternidad)

Sans doute l'expression la plus incisive de la caractéristique qu'on s'apprête à soulever ici est celle des travaux de Fréderic Worms. On lui doit, notamment, la définition sommaire, mais précise, de ce qu'il nomme "le moment du vivant" (WORMS, 2013/4, p. 120). Notre article, à plusieurs égards, s'inscrit ainsi dans la perspective qu' il prône, et il viendrait ajouter à sa théorie, plutôt occupée par Bergson, Foucault, Canguilhem et d'autres, une précision supplémentaire. Bien qu'il en soit tout à fait conscient, comme d'ailleurs il l'exprime, on trouve que cet aspect ne reçoit pas toute l'attention que, à mon avis, il mérite.

Dans ce sens, il s'agit pour nous ici, et ailleurs, de présenter une tension particulière de l'œuvre de Derrida vis-à-vis de ce "problème du vivant" qui est soulevé par M. Worms. Plutôt qu'un désaccord, il s'agirait pour nous d'approfondir la question de la vie chez cet auteur, afin de redimensionner, sous une nouvelle perspective, ce qu'il en est de la vie dans la philosophie française au XXème. À 
savoir, la lumière, peut-être singulière, que l'œuvre de Derrida peut jeter sur ce problème. Le même qui a occupé, en gros, tel qu'exprimé par Worms, toute la philosophie française dans ce siècle.

Deux choses cependant doivent être soulignées avant notre démarche, puisqu'elles jalonnent le cadre où s'inscrit notre article et synthétisent fort bien l'incisivité de l'œuvre de Derrida. Ayons présent à l'esprit, donc, l'affirmation assez simple mais très opportune de Frédéric Worms:

Telle est la question, l'alternative, devant laquelle semble en tout cas nous placer un fait trop peu remarqué, mais qui suscite la surprise, et dont nous partirons ici: le fait que la "dernière" publication (deux d'entre elles sont d'ailleurs “posthumes") de Michel Foucault (en 1985), de Gilles Deleuze (en 1995), de Jacques Derrida (en 2005), porte sur la vie. (WORMS, 2011, p. 349)

De toute évidence, fait effectivement peu remarqué, la chose qui rassemble dans une même "école" (si diversifiée, pourtant), ces trois penseurs, beaucoup plus que tant d'affirmations vagues sur une (non-)philosophie ou quelque post-modernité post-structurale, ou même une transition esthétisée avec la "littérature", c'est bien la question de la vie qui se module sous le vitalisme ou la biopolitique (et l'écriture, bien entendu). Ce qu'est cette question de la vie, voilà ce qu'on proposera au fur et à mesure dans cet article. Quoiqu'il en soit, et au-delà de cette remarque qui pourrait sembler casuistique, si ce n'était pas, justement, un symptôme, une parfaite exemplification, l'autre affirmation assez nette de Worms, avec laquelle nous présentons ici notre position, est la suivante:

Il semble tout d'abord que le problème de la vie qui y est à chaque fois saisi comme une polarité vitale, intérieure à la vie, éclaire en retour les autres problèmes, les autres tensions, qui caractérisent le "moment philosophique des années 1960" que ces pensées ont parmi d'autres contribué à constituer. (WORMS, 2011, p. 367)

Si la philosophie, comme on va l'aborder, justifie une proéminence de la logique sur la mathématique, ergo la science, c'est 
parce que comme l'aura montré de façon inlassable Derrida, le logos est vivant. Le logos, y compris la logique spéculative de Hegel, comme dirait Hyppolite, "est à l'ancienne logique formelle ce que le vivant est au cadavre" (HYPPOLITE, 1953, p. 122). Son autorité ainsi dépasse les cadres de la morale, de la politique, de la sociologie, bref, de tout ce qu'on nomme, souvent trop vite, les "apparences". Il s'agit là d'une autorité vitale, qui se justifie donc par la vie, ce qui érige le sens de l'idée de nécessité ou d'une chose impérative. Autorité dont le droit, et l'irréductibilité de celui-ci, en somme, comme disait Rousseau, s'écrit d'une encre plus forte que toute autre - dans le cœur-même des hommes, se plaît-il à affirmer (DERRIDA, 1967a, p. 29).

Il fallait donc mentionner, en passant, que quelques années avant la parution de L'histoire de la sexualité, dont le premier tome date de 1976, déjà en 1974 Derrida publiait cette œuvre très difficile mais autrement importante, intitulée Glas. Sommairement, dans un texte publié avant sa parution, Derrida le décrivit comme portant sur la "différence sexuelle dans l'économie dialectique spéculative" (DERRIDA, 1972a, p. 89) ${ }^{1}$.

À cela il fallait rajouter, entre autres, ce passage fort intéressant, dans La dissémination, livre de 1972 (p. 58), où Derrida enquête sur ce mot de Novalis, le logos spermatikos et la métaphore séminale de la connaissance, à laquelle Derrida (1974, p. 35) revient, justement, dans Glas. Chose qui, comme Derrida l'indique, était abordée par Bachelard (et on y trouve les traces de cette tradition épistémologique autour de la question de la vie) lorsque dans La formation de l'esprit scientifique il enquêtait sur cette analogie entre le sperme et l'or. Comparaison, peut-être, plus capitale. Sans doute ces questions sont analogues aux énoncés de Foucault, dans le quatrième tome de son Histoire de la sexualité, Les aveux de la chair, lorsqu'il parle d'une physiologie du logos (FOUCAULT, 2018, p. 47).

Logos spermatikos qui d'ailleurs était toute une pièce centrale des apologies de Saint Justin, qui avec Saint Jean a certainement trou-

1 La citation complète: "Dans un travail en préparation sur la famille de Hegel et la différence sexuelle dans l'économie dialectique spéculative, nous ferons apparaître l'organisation et le déplacement de cette chaîne qui rassemble les valeurs de nuit, de sépulture, de loi divine - familiale - féminine comme loi de la singularité". La première version de ce texte, "Le puit et la pyramide", parut en 1971, même année où Derrida donnait le séminaire intitulé "La famille de Hegel”, texte depuis lequel il composa Glas (DERRIDA, 1974). 
vé du goût à ce mot grec de Logos. Justin qui se plaisait à comparer, par ce goût même, et avec toute la tranquillité du monde, Socrate à Jésus. Justement alors que, dans cette même apologie, il est souvent question de mariage, naissance et baptême - et dont certains extraits sont cités, notamment par Foucault, dans ce livre tout récent qu'on citait en haut ${ }^{2}$.

L'autre aspect de La dissémination, qu'il faut sans doute avoir présent à l'esprit, sous peine d'ignorer l'interpénétration entre Foucault et Derrida, réside dans le fait que, en effet, ce dernier est le premier à remarquer la chose suivante:

Même si nous ne voulions pas ici nous laisser conduire par le passage facile qui fait communiquer entre elles les figures du roi, du dieu et du père, il suffirait de prêter une attention systématique - ce qui à notre connaissance n'a jamais été fait - à la permanence d'un schème platonicien qui assigne l'origine et le pouvoir de la parole, précisément du logos, à la position paternelle. Non que cela se produise seulement et par excellence chez Platon. On le sait ou on l'imagine facilement. Mais que le "platonisme", qui installe toute la métaphysique occidentale dans sa conceptualité [je souligne], n'échappe pas à la généralité de cette contrainte structurale, l'illustre même avec un éclat et une subtilité incomparables, le fait n'en est que plus signifiant. (DERRIDA, 1972b, p. 86)

Si on y ajoute le fait que toute la différence entre le logos (la voix) et l'écriture (la trace), celle-ci soumise au phonocentrisme, dérive du fait que, avec sa tension séminale, le logos est un vivant, et que donc l'écriture, comme dans la métaphorique rousseauiste (et bien d'autres), est signe de mort, tout comme le sera l'entendement formel pour Hegel (cette mathématique kata medemian symploken), alors on mesure mieux quelques traces du surgissement de ce concept de Derrida, le phallogocentrisme. En effet:

2 Pour d'autres précision sur l'origine du terme de Logos spermatikos, Michel Fédou a publié un article sur le sujet dont les commentaires importent sur cette connaissance qui se produit par fécondité et insémination (FÉDOU, 2009). Parmi d'autres, Foucault cite ce passage, fort particulier, où Justin affirme: "[...] la procréation est pour nous la mesure du désir”, (FOUCAULT, 2018, p. 11), comme si c'était bien cet élément, qui va avec la création (pro), la mesure, intrinsèque, du désir d'engendrer. 
Le statut de cet orphelin [l'écriture, la machine, la calculability] qu'aucune assistance ne peut prendre en charge recouvre celui d'un graphein qui, n'étant fils de personne au moment même où il vient à inscription, reste à peine un fils et ne reconnait plus ses origines: au sens du droit et du doit. À la différence de l'écriture, le logos vivant est vivant d'avoir un père vivant (alors que l'orphelin est à demi-mort), un père qui se tient présent, debout près de lui, derrière lui, en lui, le soutenant de sa rectitude, l'assistant en personne et en son nom propre (DERRIDA, 1972b, p. 87)

Quoique cela doive à la psychanalyse et à Freud - chose qu'on peut sérieusement mesurer ou, au contraire, rejeter en bloc avec le mépris désormais courant, et souvent grossier -, ces prises de position de Derrida sur la question de la différence sexuelle sont désormais d'une importance élémentaire, tant historique que philosophique. On ne le nierait qu'en vain. Importance qu'on souligne et qui contrarie, par-là même, tant de critiques maladroites qui parfois sombrent dans le ridicule d'accuser le fait (c'en est un) que Derrida n'a jamais discuté la pensée de Simone de Beauvoir. Comme si la chose la plus correcte et pertinente, et sans doute intéressante, si c'était au moins le cas, ne consistait plutôt à se demander pourquoi aucun de ces grands philosophes contemporains ne l'aient fait non plus $-y$ compris, donc, Derrida.

Ce sera un peu à la façon dont tous les deux, Derrida et Foucault, parlent de ce résidu ou de cette incomplétude de la biologie, qui pour cette raison doit faire place à une philosophie de la vie, qu'on verra le jeune Derrida construire son objection à Husserl vis-à-vis de la finitude et la problématique relative à la différence entre l'infini et l'indéfini ${ }^{3}$.

3 L'avis de Derrida sur cet aspect de la biologie peut être retrouvé notamment dans Otobiographies, p. 41-42. Celui de Foucault est recueilli dans ses Dits et écrits, III, p. 440, dans le texte intitulé "La vie: l'expérience et la science". Texte qui est, non par hasard, celui auquel Worms faisait référence, plus haut, à savoir le dernier texte de Foucault et qui aborde, encore une fois, la vie. Nota bene: Le même texte peut aussi porter le titre "Introduction par Michel Foucault", puisqu'il fût d'abord publié comme introduction à l'édition anglaise de Le normal et le pathologique de Georges Canguilhem. À la même époque, Foucault l'envoyait aussi à la Revue de métaphysique et morale, avec l'autre titre, 
Fait très remarquable dans la construction historique de cette problématique, c'est le titre très important de Bolzano sur Les paradoxes de l'infini. Nulle surprise si ce livre commence fort justement avec une critique de Bolzano à Hegel, soulignant précisément le dédain de ce penseur vis-à-vis de l'infini des mathématiciens, et accusant, par-là même, cette indéfectible intromission du philosophe dans les mathématiques, et qui concerne, précisément, l'idée de l'infini (BOLZANO, 1991, p. 45-48). À plusieurs égards, c'est l'impossibilité - technique, disons - de purifier ce concept (l'infini) de ses aspects ontothéologiques qui empêchera toujours la réalisation mathématique de la mathématique. Et c'est alors que l'infini laisse ouverte la porte par où l'autorité philosophique doit atteindre le raisonnement. Hilbert le savait peut-être mieux que personne. L'ambition de son finitisme, d'ailleurs, ne découlait probablement pas d'autre chose. À savoir, débarrasser la répétition mathématique des enchaînements philosophiques sur l'infinité.

Ce concept d'infini est très particulièrement la pierre de touche de ces problématiques. Si l'on dit parfois que c'est d'abord avec un livre sur la philosophie des mathématiques que Derrida débute sa carrière ${ }^{4}$, c'est parce qu'en effet toute sa pensée, et particulièrement celle qui porte sur la question de la finitude entre Hegel et Heidegger (et Levinas et Blanchot), y trouve une racine remarquable, bien qu' elle soit autrement complexe. Le concept d'infini, disait-on, donc, est effectivement un objet de discussion privilégié pour saisir la complexité à laquelle on se réfère ici.

Que Badiou, par ailleurs, dans un envoi à Derrida, manuscrit dans son livre Le nombre et les nombres, 1990, écrive: "À Jacques Derrida, cette façon co-variante de soustraire le Nombre à la juridiction de l'école analytique"s, voilà une chose qui servirait aussi à enlever l'animosité du débat, si c'en est un, ainsi que redimensionner les inquiétudes de la déconstruction. Il peut être frappant de lire,

pour qu'il soit publié en français dans un numéro d'hommage à Canguilhem (il s'agit du n. 1 de la 90ème année, paru en 1985).

4 Par example: "As surprising as this may sound to today's readers, Derrida's first book was a project in the philosophy of mathematics." (BARING, 2011, p. 147).

5 La copie du livre se trouve dans les archives de la Bibliothèque personnelle de Derrida à Firestone Library of Princeton University. 
cependant, dans un autre envoi, de 1982, dans La théorie du sujet, la chose suivante: "À Jacques Derrida, d'une proposition que pour dé-construire il faille le lieu où détruire"6.

La position de Badiou dans la philosophie française sur cette question de la vie, comme souligne Worms, est sans doute singulière (WORMS, 2013/4, p. 125). Ce n'est pas un hasard puisqu'il en est, quand même, très conscient. Notre article ici, en repérant l'influence de Cavaillès sur Derrida, sans doute servirait à corroborer l'affirmation suivante de Badiou. La soutenir, quand même, cela serait une toute autre chose. Cependant, ce qui est certain c'est que jamais on ne pourra, avec la désinvolture de Badiou du moins, répéter cela sans une sorte d'étonnement sur le visage, fait qui avoue l'arrière-goût de cette destruction. C'est-à-dire, il y va bien de ce qu'on essaie ici de présenter comme cette autorité qui n'est ni plus ni moins que philosophique, c'est-à-dire, une autorité logique:

Penser l'existence sans finitude. Tel est l'impératif libérateur [c'est quand même assez éloquent], qui dissocie l'exister de son épinglage au signifiant ultime de la soumission, qui est la mort. [...] À quelles conditions l'existence, la nôtre, la seule que nous puissions attester et penser, est-elle celle d'un Immortel? C'est, Platon et Aristote étaient au moins d'accord sur ce point, l'unique question dont on puisse dire qu'elle relève de la philosophie, et d'elle seule [je souligne] (BADIOU, 2006, p. 284).

Le débat avec Badiou restera longtemps ouvert. On le retrouvera, forcément, ailleurs. Mais oublions, pour l'instant, la finitude: l'histoire de l'infini (s'il y en a), pourtant, suscite des problèmes qu'on aura déjà rencontrés. Déjà Oskar Becker synthétisait ce litige: "[Pour Leibniz] le problème mathématique fondamental est celui de l'essence et de la maîtrise de l'infini" (BECKER, 2018, p. 14). Autrement dit, à croire Badiou, le problème fondamental de la mathématique est celui de permettre (ou reconnaître?) l'existence du philosophe. Mais par quelles ruses le philosophe l'oblige-t-il?

6 La copie du livre se trouve dans les archives de la Bibliothèque personnelle de Derrida à Firestone Library of Princeton University. 
Hilbert, lui aussi, avait un regard lucide sur la chose, d'après l'affirmation qui nous arrive par le texte de Cavaillès: "[L'infini est le] Seul moment extra-mathématique du raisonnement, mais décisif, chute dans ce que Hilbert appelait le transcendantal, ou réalisme psychologique" (CAVAILLÈS, 1962, p. 126). Si, par un autre côté, on sait bien qu'avec la contamination et le pharmakon la philosophie de Derrida tient pour cible, tout d'abord, l'origine simple et l'idée de pureté chez Husserl (mais pas seulement), c'est parce que ce que signifie ce pur, cela est très simple: "pureté de l'idéalité, c'est-à-dire l'ouverture de la répétition du même à l'infini” (DERRIDA, 1967b, p. 59).

Seulement alors comprend-on ce qui se passe pour Derrida avec la finitude. Notamment là où Hegel et Heidegger, tout différents qu'ils soient entre Aufhebung et vorlaufende Entschlossenheit ${ }^{7}$, se rejoignent néanmoins. Le problème est bien dans ce que Hegel nommait la certitude sensible (HYPPOLITE, 1953, p. 13-21). Ou dans la définition, philosophiquement baroque, qu'en donne Heidegger: "Der Tod als Ende des Daseins ist die eigenste, unbezügliche, gewisse und als solche unbestimmte, unüberholbare Möglichkeit des Daseins." (HEIDEGGER, 1967, \$52, p. 258). Cependant, et on revient au problème de l'articulation complexe entre ces philosophes qui se disent continentaux ou analytiques: "pour penser la finitude, vous avez déjà pensé l'infini.” (DERRIDA, 1990, p. 388). Reste à savoir si l'inverse n'est pas tout aussi vrai.

Une autre répercussion importante de ces questions, à côté de laquelle passent tous ceux qui avec dédain les considèrent comme une simple matière d'improvisation poétique et existentielle, c'est que cette même discussion, d'une façon tout à fait notable, se déroulait entre Turing et Gödel. Repérer en quoi l'idée d'infini mathématique articule une conception naturaliste de la vie, à suivre Worms, ce serait un tout premier pas pour philosopher en français. L'idée de reproduction (mimèse, récursivité, itération, etc.) de la conscience humaine par ces machines était en effet très solidement posée par Turing. Ce n'est pas un hasard si c'est par l'allégation du théorème de l'incomplétude que Gödel accusera "A philosophical error in Turing's

7 Il s'agit de la "résolution anticipante" du Dasein. Heidegger en revient à plusieurs reprises, notamment p. 302-328 de Sein und Zeit. 
work". Sans doute fallait - il mieux le décrire que ce que l'on fait ici, faute de temps et d'espace, comme toujours, pourtant, le fait est là: l'implication majeure du théorème de l'incomplétude de Gödel est celle de remarquer la nécessité du rapport entre infini et fini pour toute relation d'objets - entreprise, c'est le moins qu'on puisse dire, abyssale. Son argument, et cela ne pourrait pas se passer autrement, reviendra justement sur la nécessité incontournable pour tout calcul ou raisonnement humain de présupposer l'idée de l'infini, dont les machines seraient censées se passer, puisque, comme disait Hilbert, à ne pas le faire on retombe dans le transcendantalisme. Pourtant c'est bien le théorème d'incomplétude de Gödel qui a mis fin aux expectatives formalistes de ce mathématicien, faisant chuter l'emprise formaliste dans le transcendantalisme, ou l'axiomatique. C'est-à-dire, la philosophie existe, et par là le mathématicien doit déléguer à la logique du philosophe son autorité. L'argument de Gödel contre Turing vient préciser donc cette réciprocité que tout finitisme formel présuppose alors même qu'il entend et qu'il rêve de marcher à vide:

[...] although at each stage the number and the precision of the abstract terms at our disposal may be finite, both (and, therefore, also Turing's number of distinguishable states of mind) may converge toward infinity in the course of the application of the procedure (GÖDEL, 1990, p. 306)

La genèse, pourtant, de cet aspect, touche à son sommet probablement, on le suggère ici, dans les réflexions mathématiques de Cavaillès. Lui qui, comme Hilbert ou Hegel, Bolzano ou Husserl, était bien avisé de l'importance de ce concept, dont la controverse se déroule à travers une guerre sourde entre philosophes et mathématiciens: "L'infini est élimination de l'arbitraire: arbitraire dans le choix d'un exemple, dans l'arrêt supposé de la recherche ou de la construction." (CAVAILLÈS, 1960, p. 68) ${ }^{8}$. L'infini, en somme, c'est

8 C'est dans ce même sens, très précis, que dans un autre texte Cavaillès donnait une telle définition du nombre, par où l'on voit la pertinence réciproque du concept d'infini pour le concept de nombre, tel que Derrida parlait de l'idée infinitiste d'un simple absolu: “[...] le nombre n'est rien d'autre que l'unité de la synthèse du divers d'une intuition homogène en général' (CAVAILLÈS, 1938, p. 27). 
un autre nom de la nécessité, le il faut dont le philosophe s'arroge la prononciation, ou profession.

C'est Derrida, après, qui s'emploiera de façon plus aiguë à penser le caractère nécessairement théologique de cette assomption sine qua non des mathématiques, la modulation de ce il faut dans un on doit. Cela apparaissait déjà, de façon bien explicite, dans L'écriture et la différence: "Dès qu'il y a répétition [celle-ci est à l'infini, comme on voyait plus haut], Dieu est là [...] C'est pourquoi l'Être est le maître mot de la répétition éternelle, la victoire de Dieu et de la Mort sur le vivre" (DERRIDA, 1967c, p. 361).

Dans ce même passage Derrida cite Artaud, et l'intensité de la discussion sur l'autorité logique n'en est que plus frappante, quoique tacite: "[...] même l'infini est le nom d'un mort/ infini est le nom d'un mort/ qui n'est pas mort”. On voit bien, enfin, que la question se trouve dans le fait que, à insister avec Badiou et Meillassoux dans le renvoi de la finitude à ses enfers, toute théorie du nombre semble être aussi, à son tour, rejetée (et c'est peut-être ce qu'on a toujours cherché à faire avec son intangibilité, le platonisme de Badiou n'est que plus évident). La question, et on se tient ici avec Worms, soulignant l'importance de Derrida, serait la suivante: Qu'en est-il du logos qui ne l'est pas qu'à force d'être vivant? Et si, pour Badiou, la philosophie est pensée d'immortalité, qu'est-ce qu'un être (un logos) vivant qui soit immortel?

L'infini, et sa correspondante nécessité - spéculaire, essaie-t-on parfois d'objecter -, la finitude, est la saisie de l'unité, du simple absolu, la stigmé de l'instant radical, la verticalité même de l'un et de l'unité. Il n'est pas, et c'est sur cela que Bolzano s'emportait contre Hegel, un simple indéfini, suspension du jugement devant l'indéterminé. Car la conscience, dirait Hegel, ne se disperse pas dans la répétition vers l'insaisissable, tout élogieux qu'on puisse Être avec le Multiple. Au contraire, elle ramasse, elle cueille, après les possibilités faisables de la répétition finitiste, l'itérabilité qualifiée (non simplement quantitative, celle que Hegel appelait l'entendement formel) par le retour à soi. C'est dans le retour à soi qu'une répétition stérile et indéfinie (se prolongeant dans la mêmeté tautologique de son ennui) devient un objet, que la conscience se rapporte à elle-même, et que ce Selbst, avec son ipséité, se met du côté de l'esprit et redéfinit, par 
cet irrévocable motif du cercle et du retour, la matérialité organique, vulgaire et banale, en termes de vie. D'où la polémique de Hegel contre Kant à propos de son faux infini et le fait que Hegel alors puisse discerner entre la vraie infinitude (qui contient le fini, puisque l'infini se doit d'être saisi, atteint, donc dé-fini quelque part) et le mauvais infini qui est simple indéfinition sans mouvement de recueillement de (et dans) la conscience.

Et pourtant, c'est peut-être le seul pourtant, et peut-être qu'il n'y en aura jamais d'autre (on le dit mais on ne l'espère pas): que signifie, concrètement (a-t-on le droit de le dire ainsi?), ce mouvement de retour à soi, qui doit être assuré par le logos, qui est dès lors une chose vivante, et dont le discernement avec son autre, l'écriture, se fait par une question de vie et de mort, qui ne se discerne, à son tour, que par une question de paternité? Voilà une façon de dire les choses bien vite, de se les demander, peut-être, fût-ce à l'intérieur de ce langage du Logos spermatikos.

Pour tenir finalement aux précisions autour de la logique et de la mathématique, et acheminer quelques conclusions préliminaires sur l'importance de Cavaillès pour le jeune Derrida, on doit surtout se diriger vers cet opuscule, d'une richesse indubitable, que Cavaillès écrivit au moment même de son emprisonnement, Sur la logique et la théorie de la science. Dans ce livre, Cavaillès, d'ailleurs, semble motiver quelques-unes des critiques que Derrida fera à Husserl et, éventuellement, à une bonne partie de la tradition philosophique. Cela, même si le jeune Derrida s'occupe alors de le critiquer dans Le problème de la génèse dans la philosophie de Husserl. Cavaillès alors discute la phénoménologie de Husserl, essayant de soulever l'embarras qu'il trouve dans ce "dilemme aussi brutalement posé" (CAVAILLÈS, 1960, p. 66). Personne mieux que Derrida, pourtant, n'aura explicité le vertige phénoménologique.

Bien que le livre de Cavaillès se concentre sur une critique, le point sans doute le plus frappant de toute la démarche est surtout l'accord qu'il se verra alors contraint de concéder à Husserl. Certes, c'est aussi parce que cette critique excelle dans sa reconstruction de cette disposition fondamentale de la phénoménologie dans son rapport aux mathématiques, et qu'elle la reconnaît de façon très perspicace. Fort correctement, en effet, et comme éclairé par Derrida, 
d'ailleurs: avec la phénoménologie on assiste pour la première fois en philosophie à une posture qui - tout en affirmant la nécessité factuelle d'enchaînement, dans le monde, de l'ordre mathématique des nombres - n'entend nullement par là amoindrir la transcendantalité de ces vérités, ni se prêter à quelque scepticisme de vieille allure, si curieusement fantasmé par tant des philosophes convaincus d'en être.

On perçoit aussi déjà une préoccupation originale qui ne l'abandonnera jamais [à Husserl]: celle de l'origine concrète, dans l'expérience subjective de la perception, des significations idéales et des objets scientifiques - ici les objets arithmétiques, les nombres [je souligne, puisqu'il s'agit, bref, de la possibilité de répétition à l'infini] [...] Jusqu'alors dans l'histoire de la métaphysique, l'alternative avait été la suivante: tantôt on ne respectait pas leur objectivité et leur universalité [des nombres] - pourtant inscrites dans leur sens - et on les renvoyait à l'expérience sensible, à leur origine psychologique: c'était le geste de l'empirisme, notamment chez les philosophes anglais; tantôt au contraire, on assignait aux objets idéaux et aux vérités mathématiques qui en étaient le modèle, un lieu éternel hors de l'expérience et de l'histoire, topos noetos chez Platon, entendement divin chez les grands rationalistes cartésiens, structure apriorique de l'esprit fini chez Kant dont la notion de "formes universelles de la sensibilité pure" assurait une fonction analogue. On s'était au fond toujours abstenu devant la difficile question de l'origine: l'histoire de la métaphysique était l'histoire de cette abstention. En fait l'empirisme et le rationalisme s'étaient toujours obscurément juxtaposés et c'est leur complicité qui sera la cible de Husserl (DERRIDA, 2000, p. 71-72) .

Sans doute est-ce la puissance de cette critique husserlienne dirigée contre Kant, bien qu'elle s'avère si malheureusement insuffisante, à plusieurs reprises, qui enchaînera chez Cavaillès une réflexion remarquable sur la mathématique. Et pourtant, la question sur laquelle Cavaillès finira par reconnaître son accord avec Husserl

9 "La phénoménologie et la clôture de la métaphysique" est un texte de Derrida qui parut, en grec, en 1966. La version en français ne fut publiée qu’en 2000 dans la revue Alter. 
est bien le point qu'on essaie de mettre en perspective ici pour dimensionner l'importance de l'œuvre de Derrida. Entre les pages 53 et 55 et à travers un argument brillamment exposé, on y voit ces deux philosophes, Cavaillès et Husserl, celui-ci par des citations, fustiger la mathématique comme s'il ne s'y agissait que de la plus vaine spéculation littéraire (fait, sans doute, curieux, à plaire, cependant, plus d'un Borges).

On y assiste de façon saisissante au sommet de tout un litige intérieur à la crise des mathématiques qui s'éclate sous la plume de ce combattant, et dont le combat, d'ailleurs, ne se distingue point de la philosophie. Nombreuses sont les discussions qui semblent confluer dans ces remarques de Cavaillès. Sans doute le caractère monstrueux descellé par les géométries non-euclidiennes, entre autres choses, tellement redoutées avec toutes sortes d'arguments, jusqu'aux délires antisémites de Frege, semble être renversé par toute une histoire de la phénoménologie qui s’y affirme. Celle dont la préoccupation originale, et même originaire, comme le dit Derrida, est celle de penser la complicité entre rationalisme et empirisme, vis-à-vis du nombre.

La conclusion à laquelle aboutit alors Cavaillès, et qui, à notre avis, semble profondément motiver l'investigation que Derrida fait chez Husserl sur la finitude, nous paraît être le cas le plus extrême de tout ce qu'aura pu affirmer Worms sur la question de la vie dans la philosophie française. Ce qui donne si profondément du sens à ce fait, peu remarqué, que les publications ultimes de Derrida, Deleuze et Foucault, portent, comme on a déjà dit, sur la vie. Que Cavaillès y affirme "L'autorité de la logique a son fondement dans son rapport à la vie" (1960, p. 58), et qu'il s'agisse aussi de sa dernière œuvre, voilà une chose qu'il fallait ajouter à la remarque, très opportune, de Frédéric Worms.

Sur la logique et la théorie de la science, livre qui porte donc, lui aussi, sur la vie, semble bien établir le cadre de notre question. L'insouciance de la science, le fait qu'elle ne pense pas, dit Heidegger, est remarquablement décrit par Cavaillès - toujours avec Husserl - en rapport à la mathématique. Cet argument ratifie l'explication que Derrida donnait plus haut sur la phénoménologie, et il demeure patent chez lui comment le concept de Lebenswelt, dans ce paysage, 
est devenu impératif. Toute la philosophie française contemporaine, peut-être, $y$ trouve son fondement:

Une mathématique se constituant en science spéciale comme une fin en soi, dit Husserl, peut ne pas se soucier de ceci qu'elle est logique et méthode logique, qu'elle a une fonction de connaissance à exercer, que ses produits sont appelés à servir comme lois formelles d'enchaînements de connaissances, restant indéterminées pour des domaines de connaissance restant également indéterminées. Elle n’a pas à se soucier de ceci que le rapport à une application possible, indéterminée et toujours ouverte appartient à sa véritable signification, logique formelle... Mais le logicien philosophe doit séen inquiéter [Je souligne]. Il ne peut pas sanctionner une mathématique conçue kata medemian symploken [sans rapport à la chose, sans composition], une mathématique qui se libère de l'idée des applications possibles et devient un jeu subtil de pensées (HUSSERL apud CAVAILLÈS, 1960, p. 53).

Si donc la vie est le mot, le concept, la chose qui permet d'éclairer en retour cette "aventure philosophique", pour rappeler le titre, peut-être ironique, de Badiou, alors on comprend mieux pourquoi, entre Derrida et Foucault, la question de la sexualité est la question en question. Si Derrida a pu affirmer que la déconstruction est avant tout une déconstruction de la mort, et cela n'est pas du tout sans rapport avec un Après la finitude, comme semble, d'ailleurs, l'avoir reconnu Badiou lui-même, on remarque cependant que Derrida affirmait à la fois que la vie, non plus, ne sort pas indemne de cette déconstruction. Si toute la mathématique se plie (qu'elle le veuille ou non) à une autorité philosophique déléguée par la vie elle-même (et c'est toute l'autorité de n'importe quelle autorité), alors on ne peut penser aucun "après" la finitude sans considérer que cela implique un changement de la vie. Sans euphémisme, fallait-il le dire.

On ne sait que trop bien où se trouve la frontière, vrai fondement de la dialectique, entre la vie et la mort. Ce savoir, cependant, est un excès de savoir. C'est le caractère excessif et hors mesure du savoir. Un savoir plus que tout autre savoir. Et le savoir alors est intrinsèquement la corruption de ce que le savoir sait. Toute 
démesure y tient racine. Transiger avec cette démesure, cette chute dans l'infini comme accusait Hilbert, voilà où Cavaillès et Husserl semblent fonder la philosophie. L'atavique idée de la solitude philosophique, sa conscience au-delà du monde et de la vie (après la finitude?), transplantée et se recueillant par un retour et un détour à sa racine, comme le soulignait Badiou lorsqu'il y reconnaissait la tâche proprement philosophique, cela ne semble que mieux figurer le fait qu'il nous faut à chaque fois mesurer la démesure, atténuer l'excès, finir avec l'infini (ou bien infinitiser le fini?).

La philosophie tire alors son autorité de la restriction, nettement négative (mais pas seulement, pourtant, car il y va d'une dialectique), imposée aux autres savoirs. La philosophie capitalise les excès de ceux qui savent. Le fait que Husserl l'affirme vis-à-vis de la mathématique, et que Cavaillès, malgré ses critiques, le rejoint sur ce point, n'en est que plus frappant. La justification du logos est celle d'être vivant, de Platon à Hegel. Il se justifie parce que la démesure qu'il est lui-même doit se tordre sur soi, et la philosophie est précisément cette contorsion ${ }^{10}$. Être philosophe alors n'est peut-être pas autre chose que la toute particulière tragédie de se savoir justifié par un monde dont l'existence, du même coup, ne peut qu'être regrettée, et souvent malgré soi-même. Reste à savoir, après cela, si on a un autre choix que, ou bien vouloir par la force de ce regret atténuer sa responsabilité, ou bien par la prise en charge de ce devoir se consoler de ce que n'est que regrettable.

En effet [dit Cavaillès], le principe de réductibilité garantit que le physique le plus physique [Il faut entendre physique au sens d'Aristote] ne soit jamais perdu de vue. [... un peu après Cavaillès reprend] La logique formelle, mathesis universalis, ou a fortiori apophantique, absorbée par les complications de son déroulement propre, peut oublier ce rapport ; [cependant] la logique de la vérité, couronnement de l'édifice, puisque le reste n'en est que préparation nécessaire mais non suffisante, rétablit les perspectives. C'est en arrivant à la connaissance que toute l'œuvre antérieure s'éclaire et que les valeurs respectives

10 De façon toute sommaire Bachelard aura exprimé assez bien ce qui se partage entre biologie, mathématique, science et philosophie: "La pensée pure doit commencer par un refus de la vie" (BACHELARD, 1963, p. 21). 
des travaux préliminaires - tentés chacun de se prendre pour fin en soi - prennent leur sens authentique relativement à l'unique fin poursuivie. [...] Mais il faut qu'il soit [l'objet] effectivement atteint pour qu'il y ait savoir, la mathématique traversée s'accomplit dans la physique, il n'y a qu'un mouvement et qu'une connaissance pour qui l'unité d'ensemble garantit la validité de chacun des moments et de leurs rapports de subordination. La nécessité est partout présente et fondée parce qu'elle est unique (CAVAILLÈS, 1960, p. 53-55).

Cavaillès lui-même semble faire signe à l'aspect final. Fin unique, in extremis, l'or de toute construction, qui se place à la tête couronnée par le vrai. Tout revient au physique, la métaphysique ne s'y trouve que pour ramener ce qui dépasse le physique au physique. Le reste, ou mieux dit, toute et chaque chose sauf celle-là, prépare, se soumet à la contorsion, et cette contorsion est une voix. Puisque "Ce n'est que par oubli de sa vocation qu'elle [la mathématique] peut prétendre se suffire à elle-même" (CAVAILLĖS, 1960, p. 53). La philosophie n'est donc pas une voix au sens où cela protégerait et garderait ses mains propres vis-à-vis de l'encre qui pourrait la saillir, mais bien plutôt parce que toute écriture se rapporte à la direction de cette voix et que la philosophie sait qu'elle écrit mieux à force de ne pas écrire. La voix n'est pas matière philosophique parce qu'elle serait moins contraignante que le contrat, et que quelque chose d'humble caractériserait la philosophie, mais parce que tout contrat recule devant elle puisqu'elle surenchérit la lettre de chaque signature, et la signature se plaît, surtout, à s'écouter. Dès qu'on le dit, ça coûte trop et ça vaut plus.

Ne pas permettre aux autres savoirs que leurs têtes tournent dans le vertige (phénoménologique) de ce qu'on ne sait que trop bien, c'est le geste toujours scéniquement martyrisé du philosophe qui se sacrifie à la performativité de son autorité, où il ramasse et diminue en soi tous les autres, les autres auteurs qui s'autorisent, surtout, de la philosophie (c'est-à-dire, de l'autorité), voire les sophistes. Que l'autorité se doive à la vie, cependant, cela n'explique pas ce qu'est une autorité. Toutes les métaphores, à ce point, versent dans le même motif du rappel, du retour, ou bien de la révolution, de ce qui doit revenir (mais surtout ce qui, bien avant de revenir, ne revient que parce que 
cela est $d \hat{u})$. Mais, où il y a revenu, on est déjà ailleurs. Ce n'est qu’à force d'aller et de venir que la distance indéfinie des pas devient une seule distance et alors malgré nous une synthèse se prépare - l'infini.

Le rappel du philosophe, ce délégué en chef de la nature, fige le monde dans l'agglutination de son excès. Il rappelle la vie à la vie. Fort probablement il est une tautologie, sans doute, mais c'est une tautologie qui a lieu d'être. Nulle chose d'autre n'explique l'ennui de l'indéfini (comme disait Hegel) qui se sursomme dans un infini (et qu'il y a une différence entre ces éléments). Tautologie, donc, à laquelle la vie donne lieu, tout comme la nature, tout comme le monde, et donc, tout comme la mathématique (ergo la science), puisqu'elle revient à la physis. Cette critique toute particulière de Derrida trouvait des exemples même chez Descartes. Lorsque le philosophe rappelle, qu'il parle de ce qui lui revient (puisque, philosophe, il est maître du devenir et du revenir), l'adresse de cet envoi est toujours une métaphore, lumen naturale, disait Descartes, et une métaphore est toujours renvoi.

Le philosophe donc délègue l'autorité à la nature pour se la déléguer à soi-même. Paradoxe du don, le présent, espèce de cadeau, donne sa propre présence. Le philosophe ne le sait que trop bien. Si ce mouvement lui investit ce droit sur tout autre savoir, c'est parce que lui, lui seul et personne d'autre - on le suppose, ou bien alors on le revendique -, il sait que le savoir peut très bien (ou mal) être en trop.

S’il y a une chose sur laquelle il ne peut y avoir de savoir, ce n'est pas ce sur quoi on ne sait rien, puisque cela n'exclut pas qu'on vienne à la découvrir, mais celle que, tout en ne la connaissant point, on ne la sait que trop bien, en trop et en excès. On sait, d'une certitude plus ferme que toute autre certitude positive, que cela, eh bien, cela on ne le saura point. Là où quelque chose de plus grand que le savoir (ou même infime) s'insinue et on ne la sait qu'à force de ne pas savoir.

Or, sans savoir qu'on ne la sait pas, il n'y a pas de lieu pour la philosophie. Cet argument socratique ne sera jamais un effet de rhétorique, aussi obstinés que soient ceux qui se complaisent à le trouver en trop. Que ce soit en trop, cela sans doute ne peut pas être objecté. Que quelque part, cependant, il y ait quelque chose en trop, que cette rhétorique, toujours en trop, désigne, voilà la seule autorité de la logique, et qu'on appelle la vie, même si elle n'est pas, pour 
autant, objet de certitude. Car c'est très bien à force de ne pas être certain que cela fait trop de force. Et qu'alors, c'est à force de savoir que l'on sait.

Le logos est donc (autoritairement) l'auteur de la mathématique, puisqu'il sait bien en quoi la mathématique est en trop. Toutes les métaphores du revenir - ou bien, d'après Cavaillès, avec l'idée d'applicabilité de la mathématique, qui désigne aussi un retour à la physis - désignent ce philosophe qui ramène les excès à la mesure de la (ou mieux, de sa) nature, et de sa vie. Non pas parce que les autres vies ne sont pas des vies (tout le contraire, précisément), ou parce que la vie du philosophe n'est pas démesurée (justement, c'est une vie qui présuppose sa démesure), mais parce que la vie désignée par la vie du philosophe est une vie qui se sait en trop, parce qu' elle sait en quoi ce savoir est en trop, elle qui ne sait que trop bien ce qu'il y a de trop dans la vie. Et cela, on ne sait qu'à force de savoir qu'on ne le sait pas. Tout commence par ne pas savoir, y compris, avec quelle emphase, la vie - c'est pourquoi la biologie fait place à la philosophie. Fait que tout le reste du savoir n'ignore que pour mieux placer - comme aurait mieux dit Borges.

\section{Bibliographie}

BACHELARD, G. La dialectique de la durée. Paris: PUF, 1963.

BADIOU, A. Logique des mondes, II. Paris: Seuil, 2006.

BARING, E. The young Derrida. UK: Cambridge Press, 2011.

BECKER, O. Leibniz. Philosophie, 2018, v.1, n. 136, p. 11-23.

BOLZANO, B. Las paradojas del infinito. México: Fenian, 1991.

CAVAILLÈS, J. Le problème du fondement des mathématiques. Paris: Hermann, 1938. . Sur la logique et la théorie de la science. Paris: PUF, 1960.

. Philosophie mathématique. Paris: Hermann, 1962.

DERRIDA, J. De la grammatologie. Paris: Minuit, 1967a.

. La voix et le phénomène. Paris: PUF, 1967b.

. L'écriture et la différence. Paris: Seuil, 1967c. 
DERRIDA, J. Marges de la philosophie. Paris: Minuit, 1972a.

. La dissémination. Paris: Seuil, 1972b.

. Glas. Paris: Galilée, 1974.

. Otobiographies. Paris: Galilée, 1984.

. Le problème de la genèse dans la philosophie de Husserl. Paris: PUF, 1990a.

. Théologie de la traduction. In: Paris: Galilée, 1990b, p. 371-394. . Du droit à la philosophie.

. La phénoménologie et la clôture de la métaphysique. Alter revue de phénoménologie, n. 8, 2000, p. 69-84.

FÉDOU, M. La doctrine du Logos chez Justin: enjeux philosophiques et théologiques. Kentron, n. 25, 2009, p. 145-158.

FOUCAULT, M. La vie: l'expérience et la science. In: . Dits et écrits. France: Gallimard, 1994. p. 429-442.

. Les aveux de la chair. France: Gallimard, 2018.

GÖDEL, K. Some remarks on the undecidability results (1972a). In: FERFEMAN, S. et al. (ed.). Kurt Gödel Collected Works, II. New York: Oxford University Press, 1990, p. 305-306.

HEIDEGGER, M. Sein und Zeit. Tübigen: Max Niemeyer Verlag, 1967.

HYPPOLITE, J. Logique et existence. Paris: PUF, 1953.

MEILLASSOUX, Q. Après la finitude: essai sur la nécessité de la contingence. Paris: Seuil, 2006.

WORMS, F. Chapitre IV Pouvoir, création, deuil, survie: la vie, d'un moment philosophique à un autre. In: MANIGLIER, P. Le Moment philosophique des années 1960. France: PUF, 2011, p. 349-368.

. Le nouveau problème du vivant et la philosophie française contemporaine. Cités, 2013, v. 4, n. 56, p. 119-131.

Jeronimo Milone. Doutorando na École Normale Supérieure de Paris, bolsista de doutorado pleno pela Capes. Sua tese se desenvolve ao redor dos pressupostos biológicos da filosofia em três conceitos principais da obra de Derrida: o infinito, o sexo e a morte. Em relação à sua pesquisa publicou recentemente na Revista Guavira Letras n. 26: "O gene literal: elementos acerca da patrogênese". E-mail: jeronimomilone@gmail.com

Recebido em: 15/09/2018

Aceito em: 20/12/2018 